\title{
Karel Rychlík
}

Un manuscrit de Cauchy aux archives de l'Académie Tchécoslovaque des Sciences

Czechoslovak Mathematical Journal, Vol. 7 (1957), No. 3, 479-481

Persistent URL: http://dml.cz/dmlcz/100261

\section{Terms of use:}

(C) Institute of Mathematics AS CR, 1957

Institute of Mathematics of the Czech Academy of Sciences provides access to digitized documents strictly for personal use. Each copy of any part of this document must contain these Terms of use.

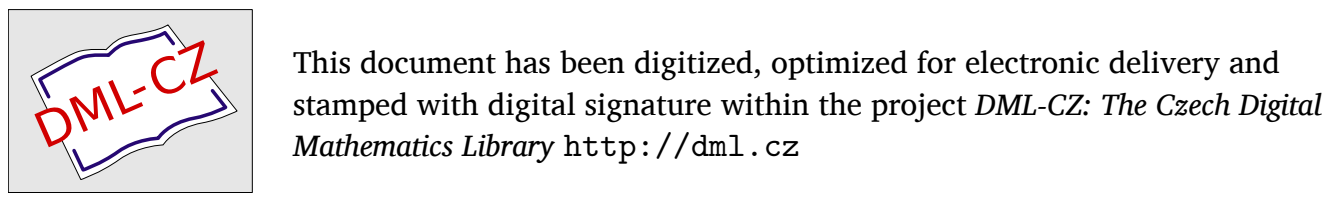




\title{
UN MANUSCRIT DE CAUCHY AUX ARCHIVES DE L'ACADÉMIE TCHÉCOSLOVAQUE DES SCIENCES
}

\author{
KAREL RYCHLÍK, Praha \\ (Reçu le 4 janvier 1957.) \\ Ecrit à l'occasion \\ du centenaire de la mort de Cauchy.
}

Après la Révolution de juillet 1830, le roi de France Charles $\mathrm{X}$ abdiqua et quitta la France. Il se rendit d'abord en Angleterre et ensuite aceepta l'invitation de l'empereur François II de s'établir avec sa cour à Prague. Il arriva dans cette ville en octobre 1832 et s'installa au château de Prague où il demeura jusqu'au mois de juillet 1836, à l'exception de courts séjours à Buštěhrad et à Teplice.

Depuis le mois d'août 1833, l'illustre mathématicien français Augustis Cauchy (né le 11 août 1789, mort le 23 mai 1857), membre de l'Académie des Sciences de Paris, était gouverneur ${ }^{1}$ ) du petit fils dé Charles X (Henri, duc de Bordeaux, comte de Chambord).

D'après le procès-verbal ${ }^{2}$ ) de la séance du 13 octobre 1833 de la Société Royale des Sciences de Bohême (SRSB), Cauchy fut élu correspondant ${ }^{3}$ ) de la SRSB (sur la proposition du professeur KULIK et du conservateur ZIPPE). Le diplôme de correspondant lui fut envoyé d'après la décision de la séance du 5 janvier 1834.

Cauchy présenta à la SRSB l'ouvrage: ,Mémoire sur l'intégration des équations differentielles" qui ne fut cependent pas publié. Sans doute, il n'exigea

1) Â ce poste, il succéda à Joachim Barrande, le célèbre géologue.

2) Les procès-verbaux de l'ancienne SRSB et d'autres écrits de cette Société se trouvent à présent aux Archives de l'Académie tchécoslovaque des Sciences.

$\left.{ }^{3}\right)$ En cette même séance C. F. Gauss fut élu, lui aussi, correspondant. 
pas le retour du manuscrit qui fut déposé aux Archives de la SRSB et passa de là aux Archives de l'Académie tchécoslovaque des Sciences. ${ }^{4}$ )

En examinant les éctris de l'ancienne SRSB j'ai pu établir à propos de ce manuscrit de Cauchy ce qui suit:

Dans une lettre du 20 avril 1836, le professeur Kulik signale que l'illustre mathématicien et membre de la SRSB présente un mémoire français avec le désir que la SRSB le fasse imprimer in $-4^{\circ}$ comme on l'a déjà fait avec le „Mémoire sur la dispersion de la lumière“. Le professeur Kulik remarque que le mémoire est d'une haute valeur, mais que c'est la SRSB qui doit décider en sa prochaine séance s'il peut être imprimé in $-4^{\circ}$. Par une très brève déclaration B. BoLzaNo se rallie à cette opinion.

- Le premier point du procès-verbal de la séance de la SRSB du $1^{\text {er }}$ mai 1836 mentione la proposition du professeur Kulik d'iprmimer la manuserit de Cauchy in-4 ${ }^{\circ}$ aux frais de la SRSB. En fait, il fut décidé de le publier dans le prochain volume des Actes de la SRSB in- $8^{\circ}$ à condition que l'auteur y consentît. Le 28 mai 1836 le professeur Kulik renvoie le manuscrit de Cauchy au secrétaire de la SRSB, Kalina, en lui demandant d'obtenir l'imprimatur de la censure. Le texte de cette demande est écrit sur la première page de la couverture du manuscrit où l'on trouve également l'accusé de réception (Präs. 30-5-1836, No 454). L'imprimatur se trouve page 72 (la dernière page) du manuscrit (2-6-1836, signé: Willmann). Au procès-verbal de la séance du 5 juin 1836 de la SRSB il est noté qu'à la lecture du procès-verbal de la précedente séance on décida, comme amendement du premier point, de ne publier le Mémoire de Cauchy que dans le tome suivant, car on possédait déjà assez de contributions pour le tome en préparation et d'autre part, en l'absence de M. Cauchy, ni le professeur Kulik ni quelqu'un d'autre ne pourrait lire les épreuves. Au mois de juillet 1836 Cauchy quitta définitivement Prague avec la cour de Charles X et les Archives de la SRSB ne fort plus aucune mention de son Mémoire.

Ce Mémoire de Cauchy parut dans les ,Exercices d'Analyse et de Physique Mathématique“ (t. I, Paris, 1840, pp. 327-384). Dans la note ${ }^{1}$ ) du bas de la page 327 on prétend que ce Mémoire ait été déjà lithographié en 1835 et au début du post-scriptum, page 384 , on précise qu'il ait été lithographié à Prague. Les „Exercices“ ont reparu dans les ,,Oeuvres complètes d'Augustin Cauchy“, ( $2^{\mathrm{e}}$ série I, t. 11, Paris, 1913); le ,Mémoire“ avec quelques suppléments se trouve pp. $399-465$, la note ${ }^{1}$ ) p. 399, le post-scriptum pp. 464-465. Mais

$\left.{ }^{4}\right)$ Le manuscrit forme une brochure in- $8^{\circ}(13 \times 23 \mathrm{~cm})$ de 72 pages, dans une couverture d'un papier un peu plus épais. Le titre „Mémoire sur l'intégration des équations différentielles. Par M. L. A. Cauchy, membre de plusieurs sociétés savantes" est inscrit sur la première page de la couverture. La signature d'Augustin Cauchy se trouve page 72 du Mémoire avec la date „Prague 1835“. Le manuscrit, bien lisible, est écrit de la main de Cauchy. 
je n'ai réussi à trouver une lithographie du Mémoire de Cauchy dans les grandes bibliothèques de Prague (bibliothèque de l'Université, de l'École Polytechnique, du Musée, de Strahov, de l'Union industrielle).

Note de la rédaction. Le lecteur trouvera des renseignements plus détaillés sur ce Manuscrit de Cauchy dans la revue „Pokroky matematiky, fysiky a astronomie“, 2, No 5, Praha 1957.

Резюме

РУКОПИСЬ КОШИ В АРХИВЕ ЧЕХОСЈОВАЦКОЙ АКАДЕМИИ НАУК

КАРЕл РЫХлИК (Karel Rychlík), Прага.

(Поступило в редакцию 4/I 1957 г.)

Во время своего пребывания в г. Прага Коши представил Королевскому чепскому научному обществу свой „,Mémoire sur l'intégration des équations différentielles". Этот мемуар не былі, однако, опубликован. Очевидно, Коши не потребовал возвращения рукописи; с тех пор она хранилась в архиве Королевского чешского научного общества и оттуда перешла в Архив чехословацкой академии наук.

Примечание редакции. Более подробное сообщение об этой рукописи читатель найдет в журнале „Pokroky matematiky, fysiky a astronomie“, 2, № 5, Прага 1957. 